\title{
Reliability and Validity of Measurement Using Smartphone-Based Goniometer of Tibial External Rotation Angle in Standing Knee Flexion
}

\author{
In-cheol Jeon ${ }^{1}$, BHSc, PT, Oh-yun Kwon ${ }^{2}$, PhD, PT, Jong-hyuck Weon ${ }^{3}$, PhD, PT, \\ Sung-min $\mathrm{Ha}^{4}$, PhD, PT, Si-hyun Kim ${ }^{1}$, MSc, PT \\ ${ }^{1}$ Dept. of Physical Therapy, The Graduate School, Yonsei University, \\ ${ }^{2}$ Dept. of Physical Therapy, College of Health Science, Yonsei University, \\ Dept. of Ergonomic Therapy, The Graduate School of Health and Environment, Yonsei University, \\ ${ }^{3}$ Dept. of Physical Therapy, College of Tourism \& Health, Joongbu University, \\ ${ }^{4}$ Dept. of physical Therapy, Division of Health Science, Baecseok University
}

\begin{abstract}
The purpose of this study was to assess the intra-rater test-retest reliability of tibial external rotation angle measurement using a smartphone-based photographic goniometer, DrGoniometer (DrG) compared to a three-dimensional motion analysis system (Vicon). The current study showed an interchangeable method using DrG to measure the tibial external rotation angle in standing knee flexion at $90^{\circ}$. Twelve healthy subjects participated in this study. A rest session was conducted 30 minutes later for within-day reliability and five days later for between-day intra-rater test-retest reliability. To assess the validity of the measurement using DrG, we used a three dimensional motion analysis system as a gold standard to measure the angle of tibial external rotation. Intra-class correlation coefficient (ICC) and the standard error of measurement (SEM) values were used to determine the within- and between- day intra-rater test-retest reliability of using $\operatorname{DrG}$ and a three dimensional motion analysis system. To assess validity, Pearson correlation coefficients were used for two measurement techniques. The measurement for tibial external rotation had high intra-rater test-retest reliability of within-day $(\mathrm{ICC}=.88)$ and between-day (ICC=.83) reliability using $\mathrm{DrG}$ and of within-day $(\mathrm{ICC}=.93)$ and between-day $(\mathrm{ICC}=.77)$ reliability using a three-dimentional motion analysis system. Tibial external rotation angle measurement using DrG was highly correlated with those of the three-dimensional motion analysis system ( $\mathrm{r}=.86)$. These results represented that the tibial external rotation angle measurement using $\operatorname{DrG}$ showed acceptable reliability and validity compared with the use of three-dimensional motion analysis system.
\end{abstract}

Key Words: DrGoniometer; Reliability; Three-dimensional motion analysis system; Tibial external rotation angle; Validity.

\section{Introduction}

Tibial external rotation is shown while the tibia is twisted outward compared with the distal end of the femur in knee flexion (Sahrmann, 2011). The measurement of tibial external rotation is quite important for clinical evaluation of the knee because a greater than normal angle of tibial external rotation is affected by patellofemoral instability (Turner, 1994), causing increased knee-valgus stress in a standing position and during gait (Turner and Smilliem, 1981). In addition, tibial external rotation may increase with maturity and influencing lateral patellofemoral impingement (Fouilleron, 2010). Previous research found that the angle of tibial external rotation with respect to the femur was significantly increased in patients with patellofemoral pain, compared with healthy subjects (Bruce and Stevens, 2004; Eckhoff et al, 1997).

Corresponding author: Oh-yun Kwon kwonoy@yonsei.ac.kr 
Since Nicol and Menelaus (1983) stated that the alignment of the lower limb structure is affected by the angle of abnormal tibial rotation; tibial external rotation is a factor to evaluate with regard to knee instability associated with knee joint pain (Bruce and Stevens, 2004).

Several studies have described different methods for measuring the angle of tibial external rotation (Mikashima et al, 2006; Taussig, 1982; Turner and Smillie, 1981; Turner, 1994; von Porat et al, 2008; Webster et al, 2010). Previous studies have shown the angle of tibial external rotation in patients with patellofemoral pain is more than $24.5^{\circ}$, while the angle in healthy subject is approximately $19^{\circ}$, as measured using a tropometer (Taussig, 1982; Turner and Smillie, 1981; Turner, 1994). Mikashima et al (2006) have documented the value of normal tibial external rotation measured using computed tomography (CT) as $10^{\circ}$ in knee extension. Sahrmann (2011) stated that the angle of tibial rotation in knee flexion at $90^{\circ}$ is between 25 and $57^{\circ}$. In addition, three-dimensional motion analysis techniques have become one of reliable and traditional ways to assess dynamic knee motion for a variety of populations (von Porat et al, 2008; Webster et al, 2010). Three-dimensional motion analysis system (Vicon) has been used to calculate the kinematics of functional knee activity such as stepping, hopping, and knee bending motions on one leg standing (von Porat et al, 2006; von Porat et al, 2008). However, these methods were too expensive, time-consuming, and complex and these features made them less accessible for clinical settings (Lee, 2003). Currently, smartphone-based photographic goniometer, DrGoniometer (DrG) (CDM S.r.l., Milano, Italy) applied in clinical practice has been increasing in popularity for a number of practitioners (Ferriero et al, 2011; Ferriero et al, 2013). Various smartphone applications with the ability to measure angles have been used to assess the range of motion (ROM) of peripheral joints in clinical settings and research (Vrtovec, 2009; Ferriero et al, 2011; Ferriero et al, 2013). These advantages of smartphones such as be- ing time-saving, expedient, and portable could be more accessible for practitioners (Jacquot, 2012). Also, their reliability has been proven through several recent studies (Al-Hadithy, 2012; Jacquot, 2012; Qiao, 2012).

To our knowledge, no studies have investigated the reliability and validity of the tibial external rotation angle in standing knee flexion at $90^{\circ}$ using DrG, compared to using a three-dimensional motion analysis system. The purposes of the present study were to (1) determine the intra-rater test-retest reliability of DrG and three-dimensional motion analysis system measurements for tibial external rotation in standing knee flexion at $90^{\circ}$ between test days and (2) assess the validity of tibial external rotation angle measurement using DrG by comparing the findings with a three-dimensional motion analysis system. We hypothesized that (1) tibial external rotation measures using both DrG and a three dimensional motion analysis system would be reliable between sessions and (2) the validity determined by correlating DrG measurement and the tibial external rotation angle, as measured by the three-dimensional motion analysis system.

\section{Methods}

\section{Subjects}

Twenty healthy male subjects were recruited by gathering a convenience sample of university students (Table 1). Inclusion criteria included: (1) normal range of motion in ankle, knee, and hip joints, (2) no musculoskeletal and neurological abnormalities,

Table 1. General characteristics of the subjects $(\mathrm{N}=20)$

\begin{tabular}{cr}
\hline \hline & ${\text { Mean } \pm \mathrm{SD}^{\mathrm{a}}}$ \\
\hline Age $(\mathrm{yrs})$ & $25.2 \pm 2.2$ \\
Height $(\mathrm{cm})$ & $74.4 \pm 5.7$ \\
Body mass $(\mathrm{kg})$ & $175.2 \pm 5.9$ \\
\hline
\end{tabular}

${ }^{a}$ mean \pm standard deviation. 
(3) no history of knee and ankle surgery. Exclusion criteria included: (1) specific ankle and knee pain such as osteoarthritis, (2) a previous history of ankle or knee surgery, (3) patella instability, and (4) symptoms of meniscal or knee ligament and ankle ligament involvement. The investigator explained all of the procedures to the subjects in detail before the measurements were taken. Subjects also signed the informed consent form.

\section{Instrument}

DrG can measure the joint angle and observe all of the related data which has been developed to perform a easier and faster measurement of joint angle (Ferriero et al, 2011). A circle highlights the center of the axis while a yellow pendulus indicated the gravity shaft. As a smartphone was placed perpendicular to the ground, the yellow pendurus showed a green color when it was close to perpendicular in the same reference position. The data on each angle calculated by placing three different markers with a gravity sensor can be stored for each subject. Each marker can be placed on the exact point of the heel (Figure 1). the picture can be taken with the gravity sensor in respect to the ground and the same height between the smartphone lens and the joint axis to make precise reference indication. In this study, the line connecting from the second toe to the heel with a molded thermoplastic tibial splint was photographed for all the subjects $1 \mathrm{~m}$ from behind when performing standing knee flexion at $90^{\circ}$.

\section{Kinematic Data}

Sixteen reflective markers of anatomical landmarks were placed over the anterior superior iliac spines, posterior superior iliac spines, lateral aspects of the thigh and leg, knee joint axis, lateral malleolus, heel, and forefoot (Webster et al, 2010). Kinematic data were sampled at the rate of $100 \mathrm{~Hz}$ with an eight camera three-dimensional motion analysis system (VICON MX system, Oxford Metrics Ltd., Oxford, UK). The diameter of reflective markers was $14 \mathrm{~mm}$, and double-sided tape was used to be attached to the skin. Before data were collected, the manufacturer's instructions were used to calibrate this system. Vicon plug-in-gait biomechanical software was used to measure the kinematic profiles. The knee flexion period was divided into three phases.
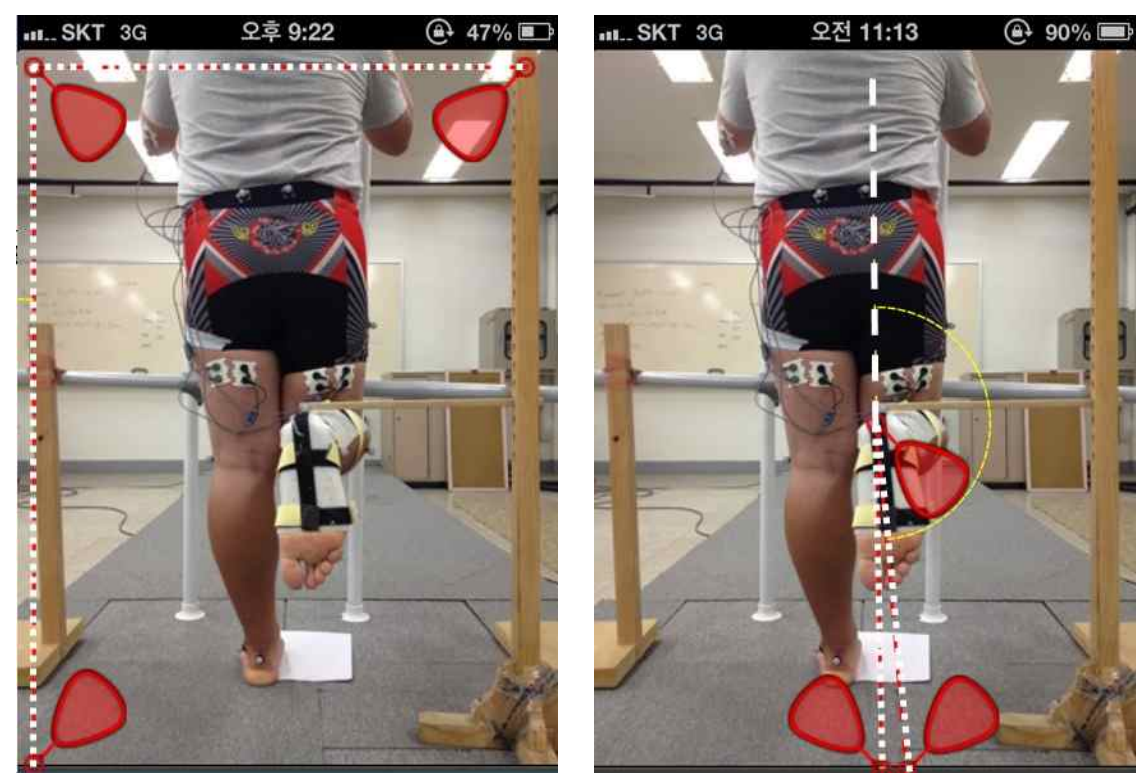

Figure 1. Three different markers with gravity sensor can be placed to calculate tibial external rotation angle performed in the frontal plane by DrGoniometer measurement. 


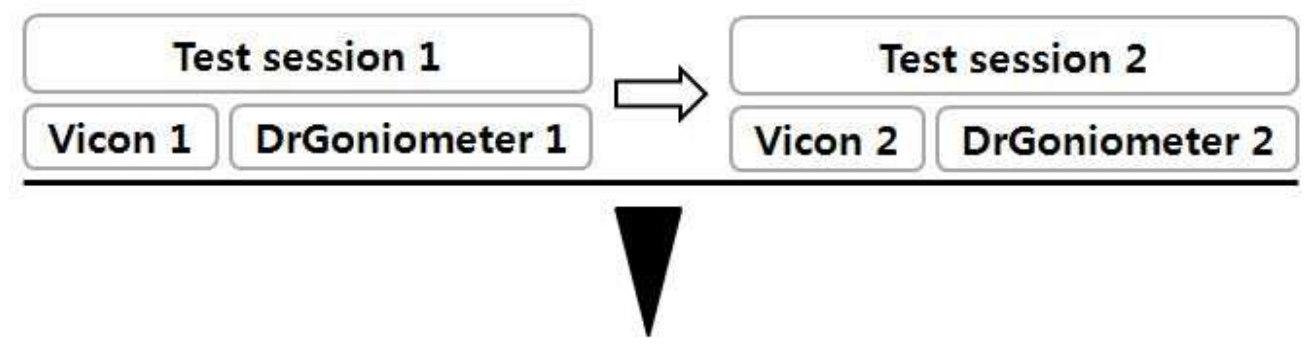

\section{\begin{tabular}{|c|c|}
\hline \multicolumn{2}{|c|}{ Test session 3} \\
\hline Vicon 3 DrGoniometer 3 \\
\hline
\end{tabular}}

Figure 2. Flow chart of the progression of measurement through the study.

Each of the three periods of knee flexion in a standing position involved placing a foot on the ground for $5 \mathrm{sec}$, being in motion for $5 \mathrm{sec}$, and being at the end range of knee flexion at $90^{\circ}$ for $5 \mathrm{sec}$. Tibial external rotation angle was measured at the end range of knee flexion at $90^{\circ}$ in the frontal plane. In addition, DrG pictures were taken at the end of the motion at $90^{\circ}$ in the frontal plane for all of the sessions.

\section{Procedures}

Subject participated in session 1 and session 2 on the same day, and session 3 was conducted five days later (Figure 1). Each subject participated in two trials only for limbs on the right-sided limbs in session 1 since there is no significant difference between left and right limbs in healthy subjects (Webster et al, 2010). Subjects were accustomed to performing the motion for sessions after practicing for 15 minutes. The examiner was familiarized with the experimental protocol to ensure standardization of the measurements using DrG and a three-dimensional motion analysis system. Since Sahrmann (2011) stated that the functional assessment of knee external rotation should be done while the participants position one of their knees at $90^{\circ}$ of flexion while standing, Each subject performed knee flexion at $90^{\circ}$ until the target bar touched the back of their calf in a standing position which allowed tibial $\mathrm{ex}^{-}$ ternal rotation to be performed in the frontal plane. Each session was started with both of their feets attached and eyes looking forward. Subjects were supported by holding two separate perpendicular shafts to minimize the weight shift. Furthermore, the ankle motion was restricted using the molded thermoplastic tibial splint (Alarm et al, 2011). Additionally, hip flexion during the tasks was limited by the fixation bar being placed in front of the thigh. Each subject was requested to sit for $30 \mathrm{~min}$ in the same place. Reflective markers were remained in the same region (i.e., session 2 was conducted after $30 \mathrm{~min}$ of resting time once session 1 was completed). After the rest period, the subjects performed the same procedure for the limbs on the right side in session 2. These two sessions were performed to determine within-day reliability. All of the subjects returned five days later and were required to perform two trials of the same procedures for right limbs in session 3 (Figure 2). The markers placement was reapplied in session 3. The data of the last session was compared with session 1 to determine between-day reliability. One examiner placed the markers on all the subjects during all of their trials. DrG and a three-dimensional motion analysis system were used together at the same time for each trial.

\section{Statistical Analysis}

Through this process, mean measurement angles 


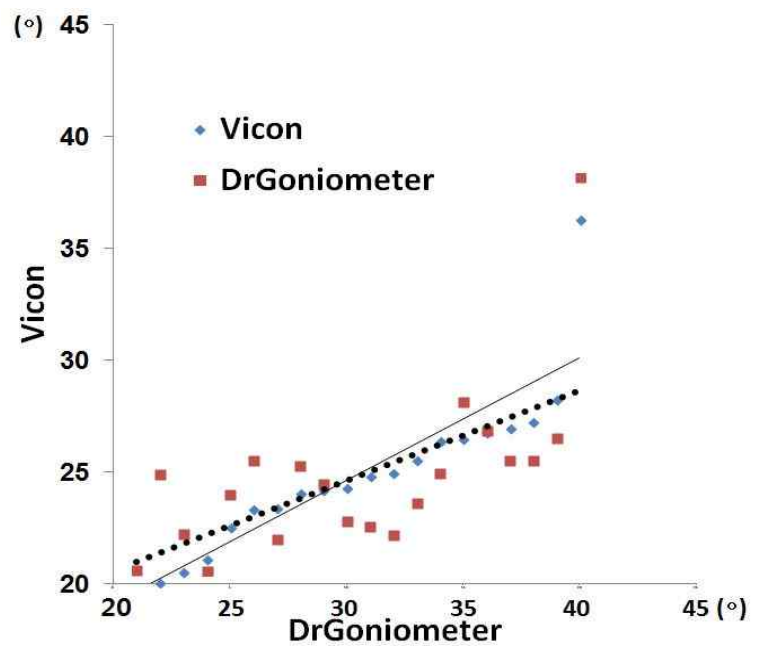

Figure 3. Scatterplot of tibial external rotation angle measurement techniques (dashed line: DrGoniometer measurement, solid line: Vicon measurement)

with standard deviations (SD) of two trials were calculated for each session. Within-day reliability was determined by the outcome of sessions 1 and 2 . Between-day reliability was determined by sessions 1 and 3. Intra-class correlation coefficients (ICC [3, 1]) and 95\% confidence intervals (CI) were used to determine the within- and between-day intra-rater test-retest reliability of using DrG and a three dimensional motion analysis system. In the interpretation, the ICC value of $>.75$ was used to indicate "excellent," .40 .75 was "fair to good," and .00 .40 was "poor" (Crossley et al, 2004). The method used to provide information on absolute reliability was the standard error of measurement (SEM), which is calculated using the following equation: [SEM=SD $\sqrt{(1-I C C)}$. Where $\mathrm{SD}=$ the sample standard deviation. The smaller SEM is expressed, the more reliable the measurement (Atkinson and Mevill, 1998). The mininal detectable difference (MDD) was calculated using the formula [MDD95=1.96 $*$ SEM $* \sqrt{2}]$ to determine the magnitude of change that would exceed the minimal error of the measurement at a 95\% confidence level (Portney and Watkins, 2009). Validity was calculated using Pearson's correlation coefficient. The correlation coefficient $(r)$ was defined as follows: >.75 was "excellent," .5 .75 was "good," $.25 \sim .50$ was "moderate," and <.25 was "poor" (Alanay et al, 2005). All statistical analyses were conducted using SPSS (ver. 18.0, SPSS Inc., IL, Chicago, USA).

\section{Results}

The intra-rater test-retest reliability of DrG and three-dimentional motion analysis system measurements was significantly good, as expected. The reliability values for the within-day ICC and between-day ICC of measurements using DrG and the three-dimensional motion analysis system are displayed in Table 2. The within-day (ICC=.93) value was higher than the between-day $(\mathrm{ICC}=.77)$ value in

Table 2. Mean and standard deviation of the tibial external rotation angle

$(\mathrm{N}=20)$

\begin{tabular}{ccc}
\hline \hline & Within day $($ session 1 and 2$)$ & Between day $($ session 1 and 3) \\
\cline { 2 - 3 } & ICC $\left(95 \% \mathrm{CI}^{\mathrm{b}}\right)$ & ICC $(95 \%$ CI $)$ \\
\hline DrG $^{\mathrm{a}}$ & $.88(.71-.96)$ & $.83(.59-.94)$ \\
Vicon & $.93(.83-.97)$ & $.77(.43-.91)$ \\
\hline
\end{tabular}

${ }^{\mathrm{a}}$ DrGoniomete, ${ }^{\mathrm{b}}$ confidence intervals.

Table 3. Mean and standard deviation of the tibial external rotation angle

(Unit: $\left.{ }^{\circ}\right)$

\begin{tabular}{cccc}
\hline \hline & Session 1 & Session 2 & Session 3 \\
\cline { 2 - 4 } & Mean $\left(\mathrm{SD}^{\mathrm{b}}\right)$ & Mean (SD) & Mean (SD) \\
\hline DrG $^{\mathrm{a}}$ & $24.8(3.7)$ & $21.7(4.0)$ & $21.6(4.1)$ \\
Vicon & $24.9(3.6)$ & $22.3(4.4)$ & $21.9(3.5)$ \\
\hline
\end{tabular}

${ }^{\mathrm{a}}$ DrGoniomete, ${ }^{\mathrm{b}}$ standard deviation. 
Table 4. Measurement error scores for tibial external rotation

\begin{tabular}{cccccc}
\hline \hline & \multicolumn{2}{c}{$\begin{array}{c}\text { Within day } \\
\text { (session 1 and 2) }\end{array}$} & $\begin{array}{c}\text { Between day } \\
(\text { session 1 and 3) }\end{array}$ & & \\
\cline { 2 - 6 } & 1.63 & SEM $^{\mathrm{b}}\left(^{\circ}\right)$ & & MDD $^{\mathrm{c}}(95)\left(^{\circ}\right)$ & $\mathrm{CV}^{\mathrm{d}}(\%)$ \\
\hline $\operatorname{DrG}^{\mathrm{a}}$ & 1.15 & 1.84 & 4.51 & 18 \\
Vicon & 1.73 & 3.19 & 19 \\
\hline
\end{tabular}

${ }^{\mathrm{a}}$ DrGoniomete, ${ }^{\mathrm{b}}$ standard error of measurement, ${ }^{\mathrm{c}}$ minimal detectable difference, ${ }^{\mathrm{d}}$ coefficient of varience.

the measurement of three-dimensional motion analysis. The between-day ICC value in DrG was lower than the within-day ICC (within ICC=.88; between ICC=.83). SEM between DrG and the three-dimensional motion analysis is displayed in Table 4. This represented the average within-subject differences for both within- and between-day sessions. Also, the between-day ICC values for DrG and the three-dimensional motion analysis were different (i.e., between-day ICC for DrG=.83; between-day ICC for the Vicon=.77), which could indicate why between-day reliability values were low, especially for the kinematic measurement with the reflective skin markers (Brochard, 2011). In addition, measurements obtained from the ICC value with $95 \% \mathrm{CI}$ of tibial external rotation, including the mean and SD values are presented in Table 2 and Table 3, respectively. A scatterplot was drawn to represent the relationship between $\mathrm{DrG}$ and a three-dimensional motion analysis measurements in Figure 3.

\section{Discussion}

The purpose of the current study was to $\mathrm{de}^{-}$ termine the intra-rater test-retest reliability and validity of DrG and three-dimensional motion analysis measures of tibial external rotation in standing knee flexion at $90^{\circ}$. We believe that the present research is the first reported study to investigate the intra-rater test-retest reliability and validity of DrG and three-dimensional motion analysis system techniques to measure tibial external rotation. A three dimensional motion analysis system was the technique in which the advanced laboratory well used to measure functional motion (Tashman et al, 2008). The measurement using DrG saves time and is convenient; thus, it is being used more frequently for clinical evaluations (Al-Hadithy, 2012; Jacquot, 2012) and research purpose with high ICC values of intra-rater and inter-rater reliability for upper limb measurements (Ferriero et al, 2011).

For intra-rater test-retest reliability, our study indicated that the value for within-day ICC was quite high compared with between-day ICC for both DrG and the three-dimensional motion analysis system. A previous study reported that within-day ICC had higher reliability than between-day ICC, which supports our first hypothesis (Webster et al, 2010). The potential reason for the higher within-day ICC values than between-day ICC values was possible adaptation in the specific tasks (Webster et al, 2010). Altered motor performance might become the reason for being more repeatable performance of subjects on the task in within-day ICC compared to between-day ICC because of the task performed on the same day. Webster et al (2010) noted that the between-day errors resulted from subtle differences in marker placement between session 1 and session 3 in a three-dimensional motion analysis system. The result reported in this investigation was quite similar to the previous studies for lower limb measurements such as level gait (Kadaba, 1989), running (Ferber, 2002; Queen, 2006), landing (Ford, 2007), and pivoting (Webster et al, 2010) even though the tasks used in these previous studies were composed of active movements compared with current study.

However, our study aimed not only to compare within- and between-day reliability using DrG and a three-dimensional motion analysis system, but also 
to provide the information that DrG is a highly reliable method to measure tibial external rotation. Even though all of the subjects were instructed to follow the same action restricted by using a parallel bar in front of their thigh to control hip flexion and by using vertical support to minimize weight shifting while standing for the same condition, a slight difference between within-day and between-day reliability was shown. So, the findings in this study might be affected as a result of the task being $\mathrm{re}^{-}$ peated on the same day which indicated the difference between within day and between day reliability. In addition, the result from a three dimensional motion analysis system might be attributed to skin marker displacement in the same way (Webster et al, 2010).

In this study, we found that DrG provided valid datas that correlated with the findings from the three-dimensional motion analysis system for the measurement of tibial external rotation with the molded thermoplastic tibial splint. Similarly, several prior studies have indicated that DrG appears to be a reliable and accurate method in clinical practice and even for the research purpose of measuring the Cobb angle in kyphosis (Jacquot, 2012), the Cobb angle in scoliosis (Qiao, 2012), and hallux valgus (Ege, 2012). Several possible explanations for our results. First, the excellent validity of DrG and three-dimentional motion analysis measurements could be explained by the knee motion being well controlled as a result of being held in the molded thermoplastic tibial splint (Alam et al, 2011). A previous study showed that tibial rotation is not accurately represented by foot rotation, and the degree of tibial external rotation is actually obscured by foot rotation (Alam et al, 2011; Costigan, 1992). The measurement using the tibial splint and the measurement performed directly on the tibia showed significant correlations at both knee external rotation $\left(6^{\circ}\right.$ overestimated in the tibial splint compared with tibia directly measurmed at 90 ) (Alam et al, 2011). DrG measurement was performed by photographing the line connecting subjects' second toe to the middle of their heel with the molded thermoplastic tibial splint from behind $1 \mathrm{~m}$ in standing knee flexion at $90^{\circ}$. For this reason, it was important to control the measurement by limiting the motion of the foot (Alam et al, 2011) and to be performed with offering a perpendicular viewpoint and centering the lens on the target angle which influenced the measurement repeatability and accuracy in photographic-based measurements (Dunlevy, 2005; Lindqvist, 1998). However, since the measurement of the tibial external rotation angle with the molded thermoplastic tibial splint using DrG is not clinically accessible, the taping method may be an alternative method to address interrelated mechanical fixation of ankle joints in clinical settings (Wilkerson, 2002). Second, another reason for the $\mathrm{ex}^{-}$ cellent validity of knee flexion at $90^{\circ}$ in standing was that the hip joint was controlled using a bar and a belt in front of the thigh to avoid unwanted hip flexion and rotation (Osternig, 1980). Osternig (1980) stated that measurement discrepancies in tibial external rotation have been attributed to the failure to control accompanying thigh motion. This implies that controlling thigh and foot motion during measurement would provide valid results for the measurement of functional motion such as tibial external rotation. This study had several limitations. First, one examiner was employed, as most of the studies progressed in the same manner. However, no clinical field can be reflected in these procedures, as the measurement can be performed by a number of therapists. Further study is required to employ more examiners to represent general clinical settings and to establish inter-rater test-retest reliability. Second, our results cannot be generalized to the patient population because young, healthy male subjects participated in this study. Therefore, further research is needed to investigate the reliability of tibial external rotation using DrG and a three-dimensional motion analysis system in individuals with knee-ankle pain and from various age groups. Third, the measurement technique using DrG for tibial external rotation 
angle cannot be generalized to the measurement for other joints angle. Further study is needed to investigate the reliability of other joints angle measurement using DrG.

\section{Conclusion}

This study demonstrated excellent intra-rater test-retest reliability and validity for the use of DrG and a three-dimensional motion analysis to measure the tibial external rotation angle in standing knee flexion at $90^{\circ}$. In summary, the findings suggested that DrG is an alternative method to three-dimensional motion analysis for measuring the tibial $\mathrm{ex}^{-}$ ternal rotation angle. Thus, measurement of the tibial external rotation angle by DrG may provide useful information for the evaluation of lower extremity kinematics and treatment outcome. Based on these findings, we conclude that 1) tibial external rotation measurements using both DrG and a three-dimensional motion analysis system obtained on the same and different days can be reliable, and 2) DrG can be a interchangeable method with a three-dimensional motion analysis system to measure the tibial external rotation angle for clinical use.

\section{References}

Alam M, Bull AM, Thomas Rd, et al. Measurement of rotational laxity of the knee: In vitro comparison of accuracy between the tibia, overlying skin, and foot. Am J Sports Med. 2011;39(12): 2575-2581.

Alanay A, Cil A, Berk H, et al. Reliability and validity of adapted turkish version of scoliosis re ${ }^{-}$ search society-22 (SRS-22) questionnaire. Spine (Phila Pa 1976). 2005;30(21):2464-2468.

Al-Hadithy N, Gikas PD, Al-Nammari SS. Smartphones in orthopaedics. Int Orthop. 2012;36(8):1543-1547.
Atkinson G, Nevill AM. Statistical methods for assessing measurement error (reliability) in variables relevant to sports medicine. Sports Med. 1998;26(4):217-238.

Brochard S, Lempereur M, Rémy-Néris O. Accuracy and reliability of three methods of recording scapular motion using reflective skin markers. Proc Inst Mech Eng H. 2011;225(1):100-105.

Bruce WD, Stevens PM. Surgical correction of miserable malalignment syndrome. J Pediatr Orthop. 2004;24(4):392-396.

Costigan PA, Wyss UP, Deluzio KJ, et al. Semiautomatic three-dimensional knee motion assessment system. Med Biol Eng Comput. 1992;30(3):343-350.

Crossley KM, Bennell KL, Cowan SM, et al. Analysis of outcome measures for persons with patellofemoral pain: Which are reliable and valid? Arch Phys Med Rehabil. 2004;85(5):815-822.

Dunlevy C, Cooney M, Gormely J. Procedural considerations for photographic-based joint angle measurements. Physiother Res Int. 2005;10(4):190 -200 .

Eckhoff DG, Brown AW, Kilcoyne RF, et al. Knee version associated with anterior knee pain. Clin Orthop Relat Res. 1997;(339):152-155.

Ege T, Kose O, Koca K, et al. Use of the iPhone for radiographic evaluation of hallux valgus. Skeletal Radiol. 2013;42(2):269-273

Ferber R, McClay Davis I, Williams DS 3rd, et al. A comparison of within- and between-day reliability of discrete 3D lower extremity variables in runners. J Orthop Res. 2002;20:1139-1145.

Ferriero G, Sartorio F, Foti C, et al. Reliability of a new application for smartphones (DrGoniometer) for elbow angle measurement. PM R. 2011;3(12): 1153-1154

Ferriero G, Vercelli S, Sartorio F, et al. Reliability of a smartphone-based goniometer for knee joint goniometry. Int J Rehabil Res. 2013;36(2):146-151.

Ford KR, Myer GD, Hewett TE. Reliability of landing 3D motion analysis: Implications for longi- 
tudinal analyses. Med Sci Sports Exerc. 2007;39(11):2021-2028.

Fouilleron N, Marchetti E, Autissier G, et al. Proximal tibial derotation osteotomy for torsional tibial deformities generating patello-femoral disorders. Orthop Traumatol Surg Res. 2010;96(7):785-792.

Jacquot F, Charpentier A, Khelifi S, et al. Measuring the cobb angle with the iPhone in kyphoses: A reliability study. Int Orthop. 2012;36(8):1655-1660.

Kadaba MP, Ramakrishnan HK, Wootten ME, et al. Repeatability of kinematic, kinetic, and electromyographic data in normal adult gait. J Orthop Res. 1989;7(6):849-860.

Lee RY, Laprade J, Fung EH. A real-time gyroscopic system for three-dimensional measurement of lumbar spine motion. Med Eng Phys. 2003;25(10):817-824.

Lindqvist B, Welander U, Mähler R. A three-dimensional photographic method for documentation and measurement of dental conditions. J Orofac Orthop. 1998;59(2):90-99.

Mikashima Y, Kimura M, Kobayashi Y, et al. Clinical results of isolated reconstruction of the medial patellofemoral ligament for recurrent dislocation and subluxation of the patella. Acta Orthop Belg. 2006;72(1):65-71.

Osternig LR, Bates BT, James SL. Patterns of tibial rotary torque in knees of healthy subjects. Med Sci Sports Exerc. 1980;12(3):195-199.

Qiao J, Liu Z, Xu L, et al. Reliability analysis of a smartphone-aided measurement method for the cobb angle of scoliosis. J Spinal Disord Tech. 2012;25(4):E88-E92.

Queen RM, Gross MT, Liu HY. Repeatability of lower extremity kinetics and kinematics for standardized and self-selected running speeds. Gait Posture. 2006;23(3):282-287.

Sahrmann SA. Movement System Impairment Syndromes of the Extremities, Cervical and
Thoracic Spines. 1st ed. Missouri, Mosby, 2011:424-425.

Tashman S, Collon D, Anderson K, et al. Abnormal rotational knee motion during running after anterior cruciate ligament reconstruction. Am J Sports Med. 2004;32(4):975-983.

Taussig G. Tibial torsion. Rev Chir Orthop Reparatrice Appar Mot. 1982;68(1):20-23.

Turner MS. The association between tibial torsion and knee joint pathology. Clin Orthop Relat Res. 1994;(302):47-51.

Turner MS, Smillie IS. The effect of tibial torsion of the pathology of the knee. J Bone Joint Surg Br. 1981;63-B(3):396-398.

von Porat A, Henriksson M, Holmström E, et al. Knee kinematics and kinetics during gait, step and hop in males with a 16 year old ACL injury compared with matched controls. Knee Surg Sports Traumatol Arthrosc. 2006;14(6):546554.

von Porat A, Holmström E, Roos E. Reliability and validity of videotaped functional performance tests in ACL-injured subjects. Physiother Res Int. 2008;13(2):119-130.

Vrtovec T, Pernus F, Likar B. A review of methods for quantitative evaluation of spinal curvature. Eur Spine J. 2009;18(5):593-607.

Webster KE, McClelland JA, Wittwer JE, et al. Three dimensional motion analysis of within and between day repeatability of tibial rotation during pivoting. Knee. 2010;17(5):329-333.

Wilkerson GB. Biomechanical and neuromuscular effects of ankle taping and bracing. J Athl Train. 2002;37(4):436-445.

This article was received March 8, 2013, was reviewed March 8, 2013, and was accepted April 28, 2013. 